\title{
Casted Wood - Tactile and Visual Aspects When Target Areas Are in Indoor Applications
}

\author{
Heidi Turunen ${ }^{1}$ \\ ${ }^{1}$ Aalto University, Department of Architecture \\ Otaniementie 14, 02150 Espoo, Finland \\ heidi.turunen@aalto.fi
}

\begin{abstract}
The most relevant senses to collect information are sight and touch, as these senses parse the world through feedback of materials, colours, shapes and structures. This paper aims to understand potentials in relation to the tangible and intangible properties of the combination of wood and cellulose nanofibrils (later CNF), when future target products are in a living environment, mostly in indoor applications. Different sizes of wood flour, sawdust or veneer-like pieces of wood have been mixed together with CNF to make material samples and designed pieces. Due to the strong hydrogen bonds and adhesivelike characteristics of $C N F$, adhesives or glue is not needed to bind materials together and, therefore, does not affect the consistency or visuality. One of the specific benefits is that the wet material does not need to be hot pressed, because during the drying period the material tightens itself. Haptic properties, such as surface temperature and texture, can be similar to wood-based products in general. Pieces can be lightweight, because the density of the material varies depending on the proportions of the ingredients. As a result, different kinds of surface structures, colours, haptic properties and visuality can be designed, varying the consistency and proportion of the materials. A mixture of wooden pieces and CNF could be manufactured into boards, but in this research, the main focus has been to understand the potentials of the casting technique to shape the material. The selected shaping method enables a new kind of method to work with wood-based materials. Although the combination of these materials has been studied, it is unknown if this has been done using methods of design research. In the future, casted wood could be utilised e.g. in indoor applications, such as furniture or architecturally related products when three-dimensional shapes are needed with natural tactile and visual appearance.
\end{abstract}

Keywords: Nanomaterials, Materials Design, Wood-based products, Architecture, Senses.

(C) Copyright 2019 Authors - This is an Open Access article published under the Creative Commons Attribution License terms (http://creativecommons.org/licenses/by/3.0).

Date Received: 2018-12-19

Date Accepted: 2019-08-08

Date Published: 2019-10-24
Unrestricted use, distribution, and reproduction in any medium are permitted, provided the original work is properly cited.

\section{Introduction}

The information on how we experience our surroundings is based on the senses, more specifically on the sense of taste, smell, sound, sight or touch. The trigger for a stimulus of a sensory experience can be as simple as colour or surface structure. Tangible material properties, such as hardness of the material, and intangible qualities, such as influence of colours when sensing thermal conditions, may be the result of conscious or unconscious perception. Those observations are collected by the senses, though the interpretations are affected, not only by pure incident, but also by past experiences and paradigms of the observer [1]. Naturally, this sensory-based feedback on the associative level may have an effect on further experiences, including reflections or conceptions of the world, and creates understanding of the surroundings and structure for the existence of a human being [2].

Material properties or sensory experiences are not necessary, stable attributes, because properties might change, for example, deliberately during the manufacturing process, even when the material stays the same [3]. Actually, a manufacturing method can be used as a tool to harness, for example, patterns or structures to interact with users in a desired way [1]. The most appropriate manufacturing or shaping method for the specific material will be defined by desired shapes, profitability of technology and properties of the material [1]. Due to this understanding, the right processing or handling methods for the material mixture are crucial.

Design plays important role especially when a specific material, the material characteristics or novel use of the material are presented in a new way [10]. 
Craftsmen generate new tacit knowledge and understanding whilst working with materials. When dealing with a relatively new material mixture, such as the mixture of wooden particles and CNF, there might not be an understanding, tacit or more scientific explicit knowledge of the material. However, combining information generated by a craftsman's method to a more scientific method, such as design research, can generate comprehensive understanding of the potentials. Those design research methods can be research through design and research by design.

When combining the two above-mentioned materials from the same kind of backgrounds, both materials are wood-based, the technical measurements and outcomes could be predictable at some levels. Visuality and processing abilities of the material might not be as clear as estimated. In addition, the processing or manufacturing method might open some new potentials. At the same time, it is possible to contemplate how to design stimuli for the senses, and to consider which of those are meaningful for future product personality, as well as how to apply those in designed material with the help of the manufacturing technique.

This research has focused on creating an understanding on how to iterate visual and tactile aspects to gain new knowledge, when designing new wood-based material for architecture. One broader goal has been to consider how a new ecological design-driven material development could create new visuality to wooden materials in future. A minor aspect in the study has been to understand valuable or desirable aspects, excluding technical properties, with this particular material mixture. Excluded from the study are technical material features, such as mechanical and thermal properties, except when valuable information about the senses can be obtained. In this case, the mould casting, which is not a very well-known method among wooden, cellulosic or nanomaterials, has been selected to be an applicable method to shape the designed material, mixture of wood and CNF.

Naturally, the focus has also been how to define, search or set limitations of moulding technique with the help of design. The mould casting, an additive method, does not create any waste at all, when comparing for example to the removing method, carving, milling or drilling, which are generally used in the wood working industry. In addition, the mould casting differs from methods traditionally used in wooden architecture, which commonly included drilling, sawing or cutting, and re-joining the shaped parts using connectors or adhesives. In architecture, mould casting is used to cast concrete structures and the type of the mould used is typically an open mould [9]. This method was also used in this study, and due to this, the surface did not need to be finished. Open moulds are open from one side [13], and with this technique water could evaporate with ease and the dry piece was easy to remove.

The remainder of the paper is organised as follows. The next section will concentrate on methods of this research and describes the research material. After that, the results chapter will present visual perceptions and tactile observations, which have emerged during making material samples and designed pieces. Then, the findings are discussed, after which conclusions are drawn.

\section{Methods and Materials}

Material samples have been implemented using research through design method at the early phase of the research. The reason for this was a need for generating new tacit and explicit knowledge on how the combination of CNF and wooden particles like to be handled, and to discover the limits of the material. As a result of this, a series of material samples was produced to understand formal, visual and tactile properties and capabilities. This first phase of the research was conducted by using moulds, patterns, colours and changing proportions in the material mixture. In addition, the focus was to implement surface structures and haptic properties, which are inherent to this specific material. Thereafter, the research by design method applied to this study aimed to visualise potentials and also to create preliminary knowledge for future product applications. The results of this second phase are designed exhibition pieces. The designed pieces disclosed new knowledge, with the help of visions, design practices and prototyping. This particular knowledge, combined with potential manufacturing processes and product applications, has also helped to more widely map visual and tactile potentials of casted wood. Material samples or designed pieces have been exhibited e.g. in the SuperMaterial exhibition, Building Centre, London, United Kingdom and the New Biomateriality Lab, Enter and Encounter exhibition, Design Museum, Helsinki, and the Nakuna exhibition during the Milan Furniture Fair, 2017.

Both outcomes, material samples and designed pieces, have been analysed using qualitative methods and tentatively compared to other existing wood-based materials and engineered wood products in the field of indoor application in interiors and architecture. 
Preliminary comparisons have been made to flat products related to indoor applications in wooden architecture. Material properties should be examined more specifically before making a comparison, e.g. concrete or plastics used in indoor applications or architecture, both can be moulded somehow. Material samples and designed pieces have been compared for aspects, such as visuality of the grain size, hardnesssoftness of the material and texture or shape of the surface. Material explorations are also not limited by the decree of technical readiness.

\section{1. Combining Wood and CNF}

The definition composite means simply a combination of two or more materials, in order to achieve better material properties than these materials have separately. Nature is full of composites, including wood, which contain a combination of cellulose, hemicellulose, lignin and other ingredients. In general, when materials are combined together as composites, the adhesives are usually plastics. This combination is due to the inherent binding properties of plastic materials. Wood polymer composites (WPC) are one example where wood fibres are combined with plastics such as polypropylene (PP), polyethylene (PE) or Polylactic acid (PLA)[12]. Even bacteria (Sporosarcina pasteurii) have been tested to bind together with sand aggregates to form bricks, but large-scale production is not yet feasible due to the by-product of ammonia [8]. A sustainable option to replace harmful adhesives might be found with mushrooms, where mycelium (fungal mycelia) is grown to bind agricultural waste to resulting building boards [8]. Composites can also be made by using biopolymers, and one such polymer is cellulose [13]. In natural polymers, one of the key benefits is in the environmental friendliness of the materials, because the harm to the environment is minimal or non-existent.

Material explorations of this study were accomplished by mixing different grainsizes of wooden materials to CNF. Both ingredients have been mixed together by hand and poured into the moulds to dry. Adhesives, or other binding agents, have not been used. The flowing consistency of the material creates 3D forms that are easy to implement. The consistency of the woodCNF mixture enables use of the moulds to shape the material, because the wet material mixture slightly resembles wet gypsum or concrete mass that is firm but wet. In room temperatures, the drying takes a few days, depending on the amounts of casted materials. One of the interesting phenomena is that hot pressing is not needed to create a tight outcome. During the drying, the material tightens itself. The curing rate of the material depends on humid conditions and heat, the latter of which can speed up the evaporation process, a prerequisite for the tightening of the structure. This self-tightening effect, caused by drying and without mechanical compression or heating, saves energy. The dehumidifying must also be taken into account when designing the mould. After the cast pieces are removed from the mould, the only postprocessing that may be needed is gently sanding.

In this study, both of the materials, the wooden ingredients and the CNF, come from the same raw material source: wood. Wood raw materials undergo a pulping process, resulting in wood pulp [14]. The wood pulp can be further processed using mechanical grinding to achieve nano or micro scale particles. In addition to grinding, there are also many other ways, such as chemical or enzymatic treatment, to process pulp towards a smaller scale [4]. However, it is beyond the scope of this research to examine how the differently manufactured CNF affects the material, and the selected treatment of pulp was mechanical grinding. As cellulose is the main ingredient of wood, and CNF is manufactured from cellulose, the designed material is mostly cellulosic, a bit more than wood. Because of this, the prepared material of this research is structurally hierarchical, as it contains cellulose in a number of different sizes. In addition to the bonding capacity, the CNF seems to also increase the strength properties of the mixed materials, which can be wood and cellulose-based or concrete [5],[6]. Strength of the pure CNF is based on strong hydrogen bonds, which are created when the material dries out. These hydrogen bonds are probably created when mixing CNF to a wood-based material which contains cellulose as well. Moulding technique have been understood to work with cellulosic materials or when cellulosic materials are mixed with other cellulosic materials [7]. Related to this, so far it is not known whether design research methods have been implemented at all when combining CNF and wood dust, sawdust or other wood-based raw material. In addition, it is also unknown as to whether the combination of wooden particles and CNF have been used commercially. The main reason might still be the low production volumes and commercialisation levels of CNF and the currently unsolved resistance to moisture.

The water content of wet CNF can be somewhere between $70-98 \%$, and the shrinkage when drying is huge. Mixing dry wooden particles with wet pure CNF causes only a very low shrinkage to dried material. 
Water probably paves the way for CNF towards the inside of the wooden material, where strong hydrogen bonds are created after evaporation. However, when CNF is mixed with wooden ingredients, the change in size after drying depends on the proportions of the material mixture.

The wooden particles of the material mixture can be recycled powdered wood, newly harvested virgin wood or by-product material from sawmills, which otherwise would be burned as wooden pellets. In the current research, the wood chips, sawdust or sanding dust are recycled from the woodworking machines of the workshop in the Department of Architecture, Aalto University. Due to this, small amounts of adhesives or paints might be found among the recycled wooden ingredient. However, the amounts are low and thus unlikely to affect the visual or tactile aspects when estimating and valuating the material samples and designed pieces.

\section{Results}

This study has been based on researching how to iterate visual and tactile aspects with a mixture of wooden particles and CNF. Different visual and tactile aspects haven been framed tentatively and applied to this study with the help of a list made by Schifferstein and Wastiels when the method to manufacture is moulding technique [3]. However, some adjustments have been made. Viewed from the visual side, Glossiness has been shifted to theme Variation of Light and Shade. The reason for this is an inherent matte outcome of both materials. The latter theme also includes surface texture characteristics from a visual point of view. When considering tactile aspects, surface hardness and softness have been left outside, because those can be varied by using different amounts of the materials. In addition to Surface Texture, a new specification, Surface Geometry, has been added to present three-dimensional aspects, which the moulding technique enables. Relations and emphasis have been compiled on the visual map to increase understanding of the connections and emphasis. As presented on the visual map, visual and tactile perceptions, when sensing the material, are generally segregated, but do have in common aspects such as surface shapes or 3D textures. In addition to creating the above-mentioned knowledge, the focus has also been in the subjects where valuable and desirable information, related to visuality and tactile aspects, are possible to generate for the future product applications.

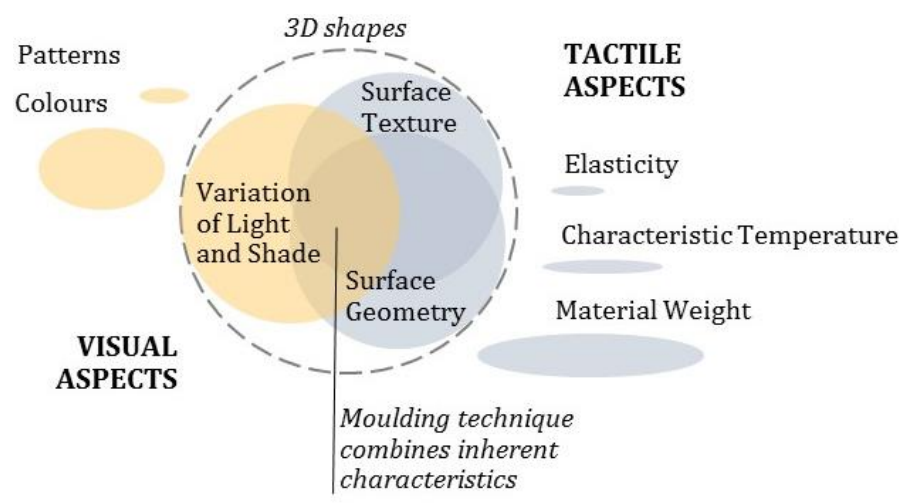

Figure 1. Initial visual map of sense perception and properties when the manufacturing technique is moulding. The size of the bubble represents how important the role of this property has when considering casted wood, whilst the aspect ratio presents how material characteristics are related to moulding. Low height of the bubble presents low benefit of this shaping technique.

VISUAL ASPECTS

COLOURS

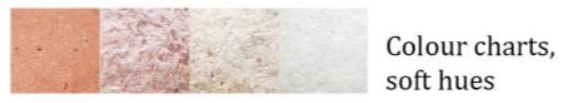

PATTERNS

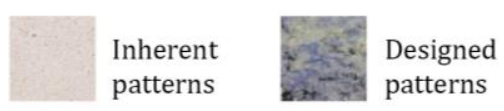

VARIATION OF LIGHT AND SHADE

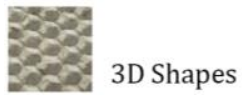

Figure 2. Material samples of visual experiments of the cast material. 


\section{TACTILE ASPECTS}

\section{ELASTICITY}

For elasticity pliable

additive is needed

CHARACTERISTIC TEMPERATURE

Sensory experience

by touching

MATERIAL WEIGHT

Possibility to make

lightweight materials

SURFACE TEXTURE

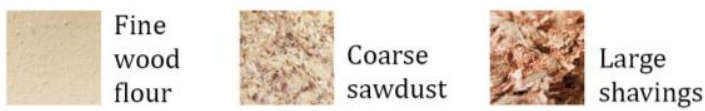

SURFACE GEOMETRY

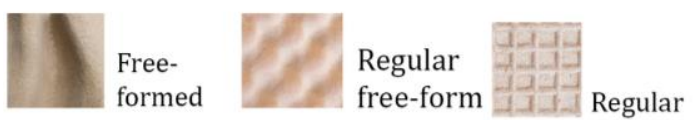

Figure 3. Material samples of tactile experiments of the cast material.

\section{1. Visual Perceptions}

The visual aspects have been studied by colouring and generating visual patterns or three-dimensional patterns in order to bring about variation in light and shadow. Particularly, the latter gets the benefit of casting technology immediately and colouring of the material in the form of a through-dyeing. Generating patterns are also created by alternating coarseness of the material, which also produces variation. All things considered, the above-listed visual modification methods can be counted to play an important role, with a different viewing distance from the material, when generating diverse visuality for this cast material.

Colours. The dyeing method, where it is possible to directly mix the colour into the raw construction material, is not very common among wooden materials. This kind of dyeing method has been used mostly when adding colourants, for example, to concrete or plastics. A more common method is coating by paint or other tinted finishing, where colouring agents have been implemented by adding layers on top of the surfaces. In this research, a potential dyeing method has been to add solid colour particles, such as natural mineral pigments or food colourants representing liquid colours. The appearance of the surface is almost similar regardless of which colouring method is used. The tones are soft, and gradations are possible to implement changing the amount of the colour. Despite the colouring method, a strong beige characteristic of undyed material, the added tone is not exactly the same as the pure colourants. This means, according to the colour theory, for example adding a hint of a blue colouring agent, the outcome looks a bit greenish. A pure green shade can be created by mixing together blue and yellow very easily, and a beige shade of the wet mass just underlines a soft and natural greenish hue. The warm beige base colour also varies slightly depending on the source and species of the wooden ingredient. Even when the outcome of pigments and liquid dyes look similar, consistency of the dyeing ingredient behaves differently inside the material. When using pigments, mouldable wooden material offers a new possibility to colour throughout the material. Instead, when using liquid colourants, the material might not be fully dyed inside. Liquid colouring agents behave as water, and when evaporation occurs, liquid colour escapes towards the outer surface of the dyed piece. This happens especially when the piece is made using fine powder, like sawdust. However, the positive aspect of integral colouring, when the piece is coloured evenly by using pigments, is that the hue does not fade away during ageing. The surface could even be renewed using sanding. Whilst evaporation brings liquid dyes to the top of the surface, the outcome can also be seen as a benefit. In this case, for example, a functional liquid ingredient for the surface could be easily added to the material at the mixing phase of the wet mass. In addition, this uneven staining can be used as a design feature for the visuality of end products.

Patterns. The appearance of a dried wood-CNF mixture is more or less homogenous and does not have apparent random or unique features, such as knots or patches, which are characteristics for timber or timberbased engineered products. However, patterns can be added to this specific material by varying the coarseness of the wooden ingredient. In addition to this inherent material-based visuality, materials can be designed. That can be achieved by adding dyes to the material mixture or adding other ingredients, such as other natural coarse 
material to the wet mixture. In this study, inherent natural patterns have been made by iterating size and natural colour of the wooden particles. Designed patterns have been created by dyeing the materials. Variations in both cases, inherent or designed patterns, have created discreet visuality, which can be noted when looking at the material close up.

Variation of light and shade. The selected manufacturing technique moulding brings positive things up to this material mixture of wooden ingredients and CNF. When CNF works like a material that increases viscosity, it makes it possible to adapt a wooden ingredient to the shape of the mould. The visual outcome of this 3D-shaped material can be various. Unfortunately, because both materials are matte, shiny surfaces are not possible to implement, and gloss is difficult to achieve. In addition, a shiny surface may not even be worth pursuing unless it would create added value to matters related to, for example, cleaning. In that case, an ingredient which provides shine might be worth adding.

\section{2. Tactile Observations}

Tactile aspects have been studied by varying material proportions and shaping the material. The most promising potentials on how to achieve haptic properties of the casted wood are the modification possibilities of the surfaces and the ability to make threedimensional surface geometries. In addition, even when dried material mixture resembles traditional wooden products, one peculiar sensory property stands out clearly. That is the lightness of the material, which is possible to achieve by adding less CNF to the material mixture. The characteristic temperature of the material does not change significantly by changing the composition of the material. Elasticity is also a stable property in this material research of the two ingredients. When considering tactile aspects, the chosen mould technology is beneficial when shaping especially surfaces or geometries. The benefit of mould technology is minor or non-existent when the focus is to vary the properties or proportions of the material mixture in case of characteristic temperature, but also in material weight and elasticity.

Material weight. Lightness of the material in general can be seen as a great benefit in certain application areas where the material's own weight does not benefit the purpose. The benefit can also be ecological and economical e.g. if there is the possibility to get strong enough material with fewer ingredients. Lightness can also be an advantage when materials are transported. In this study, proportions of wooden material and CNF have been shifted to create an understanding of how the density of the material mixture can be varied. Density of the material can alternate depending on the proportions of the ingredients, which naturally has an effect on the strength, shrinkage and weight of the material. Less CNF makes material lighter and shrinkage is lower. As CNF is a binder, a lower amount makes the material also weaker. The optional material weight - strength shrinkage ratio should be designed aiming for the purpose of the material.

Characteristic temperature. Wood is experienced as a warm material, because low thermal conductivity and warmness have been experienced as comfortable when touching wooden surfaces. Cellulose also maintains low thermal conductivity, being separated from wood and the material can be experienced as warm as well. In the material mixture of this study, apart from colourings, there are no other ingredients which would not be found in sawn timber. Because of this, casted wood feels natural and comfortable as untreated wood products. Warmth is maintained even if the material proportions of wooden particles and CNF are varied.

Elasticity. Materials can be elastic if it is an inherent or added property. If the elasticity is an added property, some of the particles of the material have elasticity as an inherent property, or combined materials together enable elasticity. Dried CNF is not an elastic material by nature, neither are the wooden ingredients which have been used in powder or chip form in this study. Hence, neither of these ingredients in this study enable elastic outcome mixed together. Although the mixture is a potential basis for adding pliable ingredients, such as glycerol to achieve elastic material with material modification.

Surface texture. A rough surface may feel uncomfortable to the hand, unlike smooth material. Some materials are inviting and want to be touched, whilst others signal keeping distance. During this research, tactility related studies have been made comparing proportions of mixed ingredients and the effect of the grain size of the dried material. The grainsize of the wooden ingredients have been tested from fine wood flour, coarse sawdust and larger shavings. Materials with very fine-sized wooden ingredient feel smooth, whilst large grain-sized pieces feel pulpy and spongy on the surface. 
Surface geometry. Three-dimensional shapes can invite touching of the material. This experience can be designed in advance by designing fascinating shapes. The moulding technique enables the making of the threedimensional shapes, repeatable forms or extremely smooth designed surfaces, manufactured at one time. In this study, the surface geometry has been studied by making regular pattern-like forms, regular free-formed shapes and complete free-form surfaces. Shaping was successful and easy to implement using the selected technique.

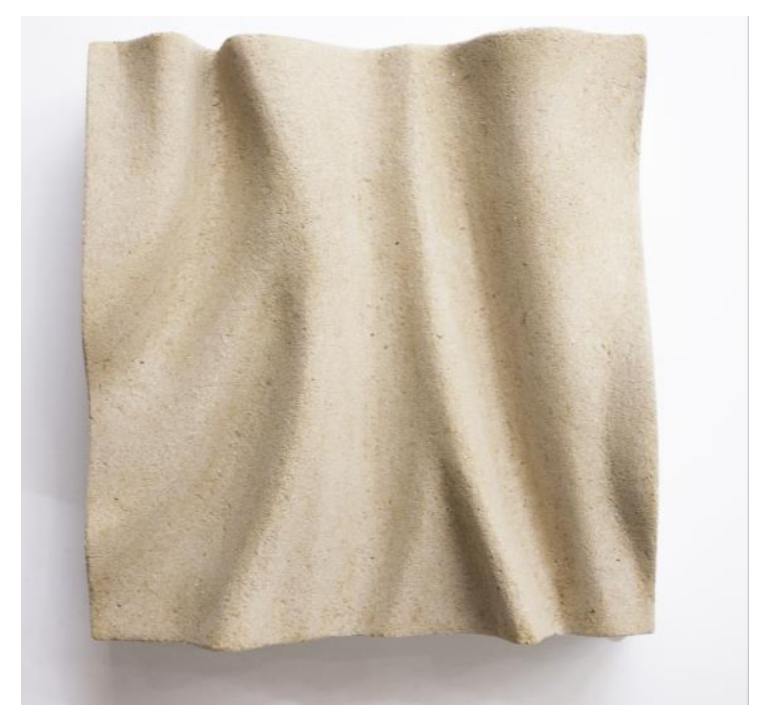

Figure 4. Designed exhibition piece, large sample 20 x $20 \mathrm{~cm}$. Photo Eeva Suorlahti.

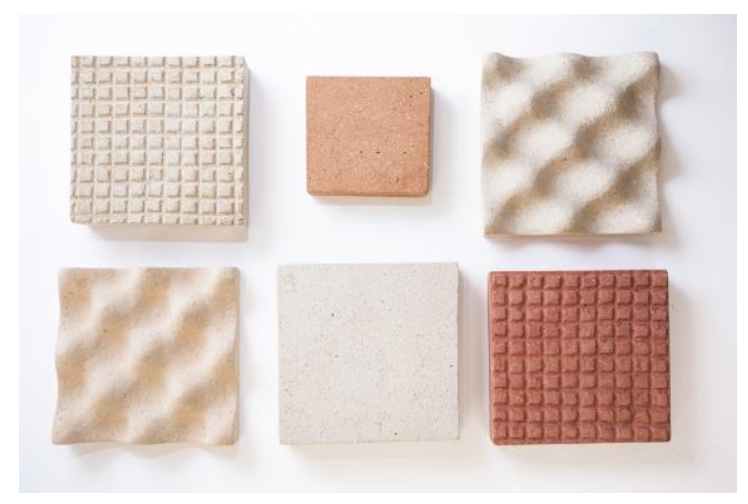

Figure 5. Small material samples and test pieces, sizes $5 \times 5$ $\mathrm{cm}$ and $10 \times 10 \mathrm{~cm}$. Photo Eeva Suorlahti.

\section{Discussion}

People have a lot of positive associations related to visual and tactile aspects in wooden products. One outcome of this research has been also to maintain highly considered properties of wood, such as naturalness of the appearance and warmness of the material. On the other hand, the aim was to deal with how to bring in and develop the new features, which moulds enable. Admittedly, the more-refined and high-tech material composition with nanoscale ingredients, the outcome in sensory level is, after all, close to the natural and unwrought materials from nature. Visuality and haptic properties of the uncoloured and flat casted wood samples do not differ considerably from engineered wood boards. The reference products could be chipboards, oriented strand boards (OSB) or mediumdensity fibre boards (MDF) and wood polymers [13], or even wood concrete [12]. However, these materials, which are coloured or three dimensionally shaped, are not reminiscent of consumer products e.g. boards sold by hardware stores, although surfaces of the MDF boards can be customised three-dimensionally afterwards at the workshops using a CNC (computer numerical control) milling machine. This may resemble the outcomes of using a wood-CNF mixture dried in the moulds; although CNC machine mills shapes in layers, whereby the surface is not completely smooth as those made in a mould. In addition, CNC milling is a material-removing technique, whilst moulding is an additive technique. Because of this, when utilising moulds, shapes could be made in a more economical way, without removal of the excess material. One great advantage of the moulding technique is that the method enables shapes, surface patterns or through dyed material at once during just one production stage. The moulds can be designed and constructed in a practice which enables casting the desired design several times using the same mould. Another great benefit is that the pieces made out of this material can be drilled, sawed, sanded and shaped using the same tools as used in the wood working industry. Furthermore, in this study, polymer-based binders are not used, but replaced with a wood-based binder, which is nanocellulose. Albeit, the binding capability of CNF needs further research, and it is probably necessary to use other additives when considering material at the product level.

Theoretically, this bio-based, environmentally friendly material mixture, combined with the moulding technique, enables shapes, textures and visuality which are common in our living environment when working 
with mouldable materials, such as clay, plastics or concrete. The focus of this research was to concentrate the early phase of the material studies by exploring variations of the materials and generating knowledge. The ratio of CNF and wood flour was similar in all the material samples representing a base which can be varied. Due to this, more detailed research is needed to understand the material applying methods of research in material science to obtain measured outcomes. In this study it turned out that the accuracy of the research outcomes can be dependant on several aspects; the amount of water in the CNF suspension, amount of dye, the humidity of the drying period and the shape of the mould taking account of the route of the water evaporation. If the material properties are further developed, and technical aspects such as reaction for moisture and stresses are researched further, all-woodbased 3D-shaped panels might be possible to implement easily. As well as controlling the material flow, using robotics could lead to 3D-printed wooden structures.

\section{Conclusion}

In this paper, the objective has been to create an understanding and new knowledge on how to iterate visual and tactile aspects when combining wood particles to cellulose nanofibrils (CNF). In addition, the present study focuses on the most informative senses, i.e. sight and touch, from a materials viewpoint. These are important as this is how the material is perceived as a starting point for both user and product personality. Mould casting has been selected as a method to shape the designed material. The main reason for this selection was the right kind of consistency of the wood-CNF material mixture.

Cellulose nanofibrils (CNF) and wood dust, or coarse sawdust, are mixed together to produce the material to be cast. A watery material combination may be poured into designed moulds, resulting in hard, predetermined shapes after the water has evaporated. The cast pieces are reproducible by reusing the moulds. Machining is not necessary to carry out, though light surface grinding may be necessary depending on the applications. Shaping possibilities when using moulds are somewhat similar to the cast materials in general. A completely wood-based material combination, without any additives, has a low environmental impact and is recyclable.

The material combination of wood-CNF has its own inherent natural tactile and visual appearance. In this study, mould-casted pieces have been observed from aspects such as colouring, patterning and variating of light and shade on the casted surface; whilst tactile properties have focused on material weight, characteristic temperature, elasticity, surface texture and surface geometry. The mould casting benefits mostly where 3D shapes are generated. For this reason, variation of light and shade, surface textures and surface geometries of the casted piece will get the most benefit from the selected manufacturing technique. In addition to the previous, promising properties, such as material weight and colouring, the material has been identified as an advantage of this material mixture in general.

In future, after a more accurate and detailed material development period, casted wood could be seen as a material for the products related to indoor applications. Those areas can be interior decoration products, sound directing designed pieces, furnishings or products related to construction industry. In addition to those areas, applications could be found also among art works, applications in sports or the toy industry, among jewellery, shoes or even disposable applications for gardens. Overall, in those areas where lightweight, through-dyed material or all-wood three-dimensionally shaped pieces will be seen to be applicable.

\section{Acknowledgements}

The research has been funded by Tekes, the Finnish Funding Agency for Innovation.

\section{References}

[1] M. Ashby and K. Johnson, Materials and design: the art and science of material selection in product design, 3rd ed. Butterworth-Heinemann, pp. 36, 103, 104, 2014.

[2] C. Norberg-Schultz, Meaning in Western Architecture. Praeger, pp. 222, 1975.

[3] E. Karana and O. Pedgley and V. Rognoli, Materials experience: fundamentals of materials and design. Butterworth-Heinemann, pp. 16, 33, 2014.

[4] V. Ballard Bell, P. Rand, Materials for Design 2. Princeton Architectural Press, pp. 9, 2014.

[5] D. K. Gulling, Manufacturing Architecture: An Architect's Guide to Custom Processes, Materials, and Applications. Laurence King Publishing, pp. 239, 2018.

[6] D. Kula, É. Ternaux, G. Hirsinger, H. Edelholt, Materiology: The Creative Industry's Guide to Materials and Technologies. Frame Publishers, Birkhäuser - Publishers for Architecture, pp. 127, 139, 191, 199, 244, 299, 2014. 
[7] S. Peters, Material Revolution - Sustainable and Multi-Purpose Materials for Design and Architecture. Birkhäuser, pp. 32, 44, 87, 2011.

[8] P. Antonelli (foreword), W. Myers, Bio Design: Nature, Science, Creativity. Thames \& Hudson, pp. 78, 106, 2014.

[9] R. Thompson, Sustainable Materials, Processes and Production. Thames \& Hudson, pp. 71, 2013.

[10] N. Lavoine, I. Desloges, A. Dufresne and J. Bras, "Microfibrillated cellulose, their barrier properties and applications in cellulosic materials: a review," Carbohydrate Polymers, vol. 90, pp. 735-764, 2012.

[11] M. Bilodeau, W. Bousfield, "Composite building product bound with cellulose nanofibers," U. S. Patent, 2015/0033983 A1, 5 Feb., 2015.

[12] S. Peters, T. Rushing, E. Landis, T. Cummins, "Nanocellulose and Microcellulose Fibers for Concrete," Transportation Research Record: Journal of the Transportation Research Board, vol. 2142, pp. 25-28, 2010.

[13] H. Döpfner, M. Ernegg, R. Bramsteidl, "Process for Producing Workpieces and Moulded Pieces out of Cellulose and/or Cellulose Containing Fiber Material,” U. S. Patent 6,379,594 B1, 30 Apr. 2002. 